\title{
Some Micellar Properties of Long-Chain Acylcarnitines
}

\author{
SAMUEL H. YALKOWSKY² AND GEORGE ZOGRAFI \\ College of Pharmacy, The University of Michigan, Ann Arbor 48104 \\ Received January 9, 1970; accepted March 3, 1970
}

\begin{abstract}
The acid dissociation constants of long-chain esters of carnitine ( $\beta$-hydroxy- $\gamma$ trimethylammonium-butyrate) above the critical micelle concentration were determined potentiometrically at several concentrations of added KCl. As the degree of protonation $\beta$ increases the apparent $\mathrm{pK}$ values decrease owing to the increased positive charge on the micelle. The difference in $\mathrm{pK}$ between the neutral (zwitterionic) micelle and the value at any given $\beta$ was used to determine the surface potential of the micelle $\Psi$ at that degree of protonation. At each degree of protonation the measured surface potential was related to the surface charge density $\sigma$ with the aid of the calculations of Loeb, Wiersema, and Overbeek for a spherical impenetrable particle. The surface potentials and surface charge densities of lauryl-, myristyl-, and palmitylcarnitine are nearly identical at a given degree of protonation and ionic strength, and, as expected, increasing the ionic strength produces a decrease in the surface potential. From the partial molal volume of each surfactant in the micelle and the calculated surface charge density it was possible to calculate the aggregation number $n$ of the micelle. Good agreement was found between the calculated values of $n$ and values obtained from light-scattering experiments at several ionic strengths and degrees of protonation.
\end{abstract}

\section{INTRODUCTION}

In a previous publication (1) it was shown that long-chain fatty acylcarnitines<smiles>CCCCCCCCC(=O)OC(CC(=O)[O-])C[NH+](C)C</smiles>

where $n=7,9,11,13$, and 15 , form micelles and that the observed $\mathrm{pK}$ of the micellar surfactant is dependent on the net charge of the micelle, the ionic strength, and the

1 This study was supported in part by the Bristol-Myers Company, Hillside, New Jersey; General Research Support Grant FR 05571, National Institutes of Health, and United States Public Health Service Training Grant DE 00204, National Institute of Dental Research, National Institutes of Health, Bethesda, Maryland.

2 Present address: The Upjohn Company, Kalamazoo, Michigan 49001. specific ions present. The present study was designed to relate the pK of the charged micelle to its surface potential, and to then calculate the surface charge density and aggregation number. Values for aggregation number obtained in this manner were then compared to those obtained by the lightscattering method.

\section{THEORETICAL CONSIDERATIONS}

Surface Potential. At any given degree of ionization $\beta$ of a micelle, the surface potential $\psi$ can be calculated from the difference between the observed $\mathrm{pK}$ at that degree of ionization and the $\mathrm{pK}$ at $\beta=0, \mathrm{pG}$, by the following equation $(1-4)$ :

$$
\psi=2.303 \frac{k T}{\epsilon}(\mathrm{pK}-\mathrm{pG})
$$

where $k$ is the Boltzmann constant; $T$ is the absolute temperature; and $\epsilon$ is the charge of an electron. 
Micelle Model. In order to determine the micelle size from the surface potential and degree of ionization it is necessary to utilize a model for the micelle. The model to be considered in this report treats the micelle as a smooth, spherical, impenetrable particle of radius, $b$. The net charge of the micelle at any degree of ionization $Z$ is assumed to be smeared uniformly over its surface, and the distribution of univalent ions near the surface is determined by a Boltzmann distribution, the distance $a$ of closest approach of an ion to the micelle center being equal to the radius of the micelle plus the radius of the electrolyte ion.

First Debye-Hückel Approximation $\left(D H_{1}\right)$. The first Debye-Hückel approximation treats all ions as point charges, and therefore $a$ equals $b$. Linearization of the PoissonBoltzmann equation leads to the following relationship between the surface charge density $\sigma$ and $\psi$ :

$$
\sigma^{\mathrm{DH}_{1}}=\frac{D \psi}{4 \pi b}(1+\kappa b),
$$

where $D$ is the dielectric constant of the solvent and $\kappa$ is the reciprocal thickness of the electrical double layer.

Second Debye-Hückel Approximation $\left(\mathrm{DH}_{2}\right)$. In the second Debye-Hückel approximation $\left(\mathrm{DH}_{2}\right)$ the size of the electrolyte ions is not ignored and Eq. [2] becomes

$$
\sigma^{\mathrm{DH}_{2}}=\frac{D \psi}{4 \pi b}\left[\frac{1+\kappa b}{1+\kappa(a-b)}\right] .
$$

If the average radius of the electrolyte ions is $2 \AA$, as is approximately the case with $\mathrm{KCl}$, Eq. [3] becomes

$$
\sigma^{\mathrm{DH}_{2}}=\frac{D \psi}{4 \pi b}\left[1+\frac{1+\kappa(b+2)}{1+\kappa b}\right]
$$

with all parameters being expressed in angström units.

Empirical Equation of Loeb, Wiersema, and Overbeek $(L)$. Loeb et al. (6) have solved the Poisson-Boltzmann equation for spheres at various surface potentials and values of $\kappa b$ by numerical integration with an electronic computer. From their results they have provided an empirical equation which can be modified to relate the surface charge density to the surface potential of a small particle,

$$
\begin{aligned}
\sigma^{\mathrm{L}}=\frac{D k T \kappa}{4 \pi \epsilon}\left[2 \sinh \left(\frac{\epsilon \psi}{2 k T}\right)\right. \\
\left.+\frac{4}{\kappa b} \tanh \left(\frac{\epsilon \psi}{4 k T}\right)\right] .
\end{aligned}
$$

Flat Plate Approximation (FP). A fourth means of treating this problem is to relate the surface charge density and potential by the analytical solution of Gouy and Chapman for a flat plate (7). Although this is not valid for spherical particles, it has been used in preview studies $(4,8)$. According to this treatment

$$
\sigma^{\mathrm{FP}}=\frac{D k T_{k}}{4 \pi \epsilon} 2 \sinh \left(\frac{\epsilon \psi}{2 k T}\right) .
$$

Geometrical Considerations. At any degree of ionization the surface charge density $\sigma$ of the micelle is related to the net charge $Z$ and the surface area $A$ of the micelle by

$$
\sigma=Z / A \text {. }
$$

The net charge on a micelle having an aggregation number of $n$ and a degree of ionization $\beta$ is:

$$
Z=n \epsilon \beta,
$$

and the aggregation number is related to the radius of the micelle by

$$
n=4 \pi b^{3} / 3 v
$$

provided that the volume $v$ of the surfactant in the micelle is independent of $\beta$.

Equations [7], [8], and [9] can be combined to give

$$
\begin{gathered}
b=(3 v / \epsilon \beta) \sigma \\
\text { or } \\
b=3 v \sigma / \epsilon \beta
\end{gathered}
$$

which is valid at any value of $\beta$.

If Eq. [1] is substituted into Eqs. [2], [4], [5], and [6] and the resulting equations are 
combined with Eq. [10], the following equations result:

and tests for purity agree well with literature values (10). Plots of surface tension vs. con-

$$
\begin{aligned}
& b^{D H_{1}}=1 / 2\left\{\frac{C v(\mathrm{pK}-\mathrm{pG})_{\kappa}}{0.434 \beta}+\sqrt{\left[\frac{C v(\mathrm{pK}-\mathrm{pG})_{\kappa}}{0.434 \beta}\right]^{2}+\frac{4 C v(\mathrm{pK}-\mathrm{pG})}{0.434 \beta}}\right\} ; \\
& b^{D H_{2}}=1 / 2\left\{\frac{C v(\mathrm{pK}-\mathrm{pG})}{0.434} \frac{\kappa}{(1+2 \kappa)}\right. \\
& +\sqrt{\left.\left[\frac{C v(\mathrm{pK}-\mathrm{pG})}{0.434 \beta} \frac{\kappa}{(1+2 \kappa)}\right]^{2}+\frac{4 C v(\mathrm{pK}-\mathrm{pG})}{0.434 \beta}\right\}} \\
& b^{L}=1 / 2\left\{\frac{C v 2 \kappa \sinh \left(\frac{\mathrm{pK}-\mathrm{pG}}{0.868}\right)}{\beta}\right. \\
& \left.+\sqrt{\left[\frac{C v 2 \kappa \sinh \left(\frac{\mathrm{pK}-\mathrm{pG}}{0.868}\right)}{\beta}\right]^{2}+\frac{C v 16 \tanh \left(\frac{\mathrm{pK}-\mathrm{pG}}{1.74}\right)}{\beta}}\right\}
\end{aligned}
$$

$$
b^{F P}=\frac{C v 2 \kappa \sinh \left(\frac{\mathrm{pK}-\mathrm{pG}}{0.868}\right)}{\beta}
$$

where $C$ is $3 \times 10^{-8} D k T / 4 \pi \epsilon$, which is equal to 0.034 at room temperature. These equations express the micelle radius in terms of five experimentally determinable parameters, $v^{1}, \kappa, \beta, \mathrm{pK}$, and $\mathrm{pG}$ for each of the treatments discussed. Using Eq. [9] then enables one to calculate $n$, the aggregation number for each case.

\section{EXPERIMENTAL}

Materials. The acylcarnitines used in this study were synthesized from DL carnitine ( $\beta$-hydroxy- $\gamma$-trimethylammoniumbutyrate) hydrochloride (Mann Research Laboratories) and high-purity $(99+\%)$ fatty acids (Applied Science Laboratories) as described previously (1). All physical constants centration show no minima, suggesting the absence of free fatty acids.

Determination of Critical Micelle Concentration. The determination of the critical micelle concentration (c.m.c.) is necessary to evaluate the relative contribution of monomeric and micellar species to the titration behavior of the long-chain acylcarnitines. This was accomplished by determining the concentration at which the break in the log concentration vs. surface tension plot occurs. The surface tensions of solutions containing various concentrations of lauryl-, myristyl-, and palmitylcarnitine were determined by the drop volume method, as described previously (1). Table I lists the c.m.c. values. for these acylcarnitines in their cationic and zwitterionic forms in the presence and absence of $0.2 M \mathrm{KCl}$.

\begin{tabular}{|c|c|c|c|c|}
\hline \multirow{3}{*}{ Compound } & \multicolumn{4}{|c|}{ Critical micelle concentration (moles/liter) } \\
\hline & \multicolumn{2}{|c|}{ Cationic form } & \multicolumn{2}{|c|}{ Zwitterionic form } \\
\hline & $\mathrm{H}_{2} \mathrm{O}$ & $0.20 \mathrm{M} \mathrm{KCl}$ & $\mathrm{E}_{2} \mathrm{O}$ & $0.20 M \mathrm{KCl}$ \\
\hline Laurylearnitine & $1.5 \times 10^{-3}$ & $7.5 \times 10^{-4}$ & $1.2 \times 10^{-3}$ & $8.5 \times 10^{-4}$ \\
\hline Myristylcarnitine & $1.5 \times 10^{-4}$ & $9.0 \times 10^{-5}$ & $1.0 \times 10^{-4}$ & $7.5 \times 10^{-5}$ \\
\hline Palmitylcarnitine & $1.5 \times 10^{-5}$ & $8.5 \times 10^{-6}$ & - & - \\
\hline
\end{tabular}

Potentiometric Titrations. Potentiometric titrations were carried out using a Beckman

TABLE I

Critical Mrcelle Concentrations of Cationic and Zwttterionic Acyicarnitines in WATER AND $0.20 M \mathrm{KCL}$ AT $25^{\circ} \mathrm{C}$ 
101900 Research pH Meter equipped with a Beckman 39167 General Purpose Glass Electrode and a Beckman 19168 Silver-SilverChloride Reference Electrode. All solutions described in this paper are well above the critical micelle concentration (see Table I) so that the contribution of the monomeric species to the titration curve is negligible.

The degree of protonation $\beta$, which in the case of the acylcarnitines is also the degree of ionization, is defined as,

$$
\beta=\frac{\left(\mathrm{RH}^{+}\right)}{\left(\mathrm{RH}^{+}\right)+\left(\mathrm{R}^{ \pm}\right)}
$$

where $\left(\mathrm{RH}^{+}\right)$and $\left(\mathrm{RH}^{ \pm}\right)$are the concentrations of cationic and zwitterionic surfactant. The value of $\beta$ is determined experimentally from the amount of titrant added and the amount required to completely titrate the sample (1). If the hydrogen or hydroxyl ion concentration is not negligible compared to that of the acylcarnitine, the condition of electrical neutrality must be considered in determining the value of $\beta$ (11). The observed pK value at any given degree of ionization, $\mathrm{pK}$, is calculated from the $\mathrm{pH}$ and the degree of ionization by:

$$
\mathrm{pK}=\mathrm{pH}+\log \frac{\beta}{1-\beta} .
$$

Molecular Volume. The partial molecular volumes of the acylcarnitines, exclusive of their counterions, were determined experimentally and by Traube's method $(12,13)$. Traube's method considers the volume of a molecule as the sum of the volumes of its component atoms; it has been used successfully for proteins $(13,14)$ and for small molecules in aqueous solution. The molecular volumes of the acylcarnitines, determined experimentally at $25^{\circ} \mathrm{C}$, were determined by pycnometry through the use of the following equation (15):

$$
\bar{v}=\frac{M}{0.6023}\left[\frac{1}{\rho}+(1-x) \frac{d(1 / \rho)}{d x}\right],
$$

where $\bar{v}$ is the partial molecular volume ${ }^{3}$ of the acylcarnitine including its counterions;

${ }^{3}$ There is no means of measuring $v$, the true molecular volume of a solute; however, the partial molecular volume $\bar{v}$ can be used as a good approximation (9).
$M$, the molecular weight of the acylcarnitine; and $\rho$ the density of a solution of weight fraction $x$. The partial molecular volume exclusive of counterions was obtained by subtracting the known partial molecular volumes of the counterions present from $\bar{v}$ (16). These latter values are based upon one of $4.5 \mathrm{cc} / \mathrm{mole}$ for hydrogen ion. The use of 0 cc/mole for hydrogen ion would result in a change of less than $2 \%$ for the volume of the acylcarnitines used in this study.

Light Scattering. Since the results of the potentiometric titrations and partial molecular volume calculations will be used to determine the size of the unhydrated micelle and since the molecular weight obtained is a weight-average value, it was decided to utilize light scattering as an independent means of determining micelle size. The need for a weight-average molecular weight is not critical since micelles are fairly monodisperse $(17,18)$; however, the need for the weight of the unhydrated particle is extremely important since a $1.5 \AA$ hydration layer could result in an error of over $20 \%$ in the micelle weight.

Turbidity measurements were made with a Brice-Phoenix Universal Light Scattering Photometer (Phoenix Precision Instrument Company) equipped with a cell table and cored jacket through which $25^{\circ} \pm 0.1^{\circ}$ water was circulated. Unpolarized light of wavelength $546 \mathrm{~m} \mu$ was used for all measurements.

All solutions were prepared with AbboLiter Sterile Water for Injection, USP (Abbott Laboratories), and were filtered through a fine porosity sintered glass funnel directly into a T-104 microcell (Phoenix Precision Instrument Company). The cell was then centrifuged at $4000 \mathrm{rpm}$ for $20 \mathrm{~min}$ to remove from the light path any suspended material that was not removed by filtration.

The refractive index increments of the surfactant solutions, used for calculating molecular weights from light-scattering data, were determined with a Brice-Phoenix differential refractometer. Light of wavelength $546 \mathrm{~m} \mu$ was used, the instrument was calibrated with $\mathrm{KCl}$ solutions, and the temperature was maintained at $25^{\circ} \mathrm{C}$.

The refractive indices of the acylcarnitines 
are independent of the degree of ionization of the micelle and of the ionic strength of the solution; these values are 0.143 for laurylcarnitine, 0.153 for myristylcarnitine, and 0.154 for palmitylcarnitine.

\section{RESULTS}

Titration Curves. The titration curves (observed $\mathrm{pK} v s$. degree of ionization or protonation) of myristylcarnitine at several concentrations of added $\mathrm{KCl}$ at $25^{\circ} \mathrm{C}$ are shown in Fig. 1. Similar curves were obtained for laurylcarnitine and palmitylcarnitine. These titration curves are independent of surfactant concentration as long as measurements are made well above the critical micelle concentration and at constant ionic strength. The ionic strength of the solutions used is calculated from the concentration of $\mathrm{KCl}$ initially present and the $\mathrm{KCl}$ formed on neutralization of the cationic surfactants. No contribution to the ionic strength was included for the micelle since it has been shown that polyions do not contribute to the ionic strength of their solutions (19). The pK extrapolated to $\beta=0$ is $4.85 \pm 0.03$ and is independent of chain length and ionic strength.

As illustrated in Fig. 1 and Table II the

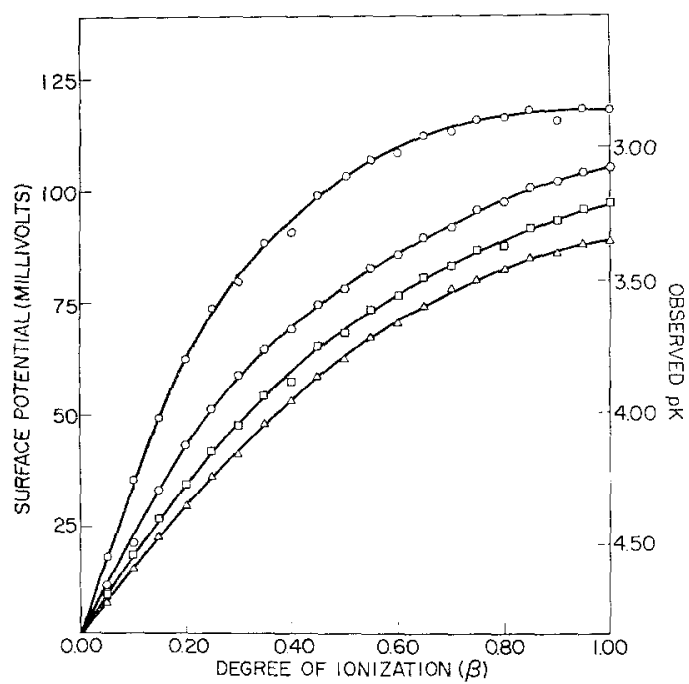

FIG. 1. Observed pK and calculated surface potential vs. degree of ionization of myristyicarnitine at several concentrations of added $\mathrm{KCl}$. $\mathrm{O}:$ no salt; $\bigcirc: 0.05 \mathrm{M} ; \square: 0.10 \mathrm{M} ; \triangle: 0.20 \mathrm{M}$
TABLE II

VALUES OF OBserved PK fOr LaURyLcarnitine, Myristylcarnitine, and PaLMitylCaRnitine

\begin{tabular}{ccccc}
\hline \multirow{2}{*}{ Solvent } & $\beta$ & \multicolumn{3}{c}{ Surfactant } \\
\cline { 3 - 5 } & & $\begin{array}{c}\text { Lauryl- } \\
\text { carnitine }\end{array}$ & $\begin{array}{c}\text { Myristyl- } \\
\text { carnitine }\end{array}$ & $\begin{array}{c}\text { Palmityl- } \\
\text { carnitine }\end{array}$ \\
\hline $0.10 M \mathrm{KCl}$ & 0.25 & 4.18 & 4.15 & 4.12 \\
& 0.50 & 3.68 & 3.72 & 3.70 \\
& 0.75 & 3.31 & 3.41 & 3.42 \\
$0.20 M \mathrm{KCl}$ & 1.00 & 3.21 & 3.21 & 3.23 \\
& 0.25 & 4.27 & 4.26 & 4.85 \\
& 0.50 & 3.80 & 3.79 & 3.80 \\
& 0.75 & 3.50 & 3.50 & 3.53 \\
& 1.00 & 3.33 & 3.35 & 3.32 \\
\hline
\end{tabular}

TABLE III

Partial Molfoular Volumes of LaUryiCarnitine, Myristylcarititine, and PalMitylcarnitine Exclusive of CounterIONS

\begin{tabular}{lcc}
\hline Surfactant & \multicolumn{2}{c}{ Partial molecular volume } \\
\cline { 2 - 3 } & $\begin{array}{c}\text { Traube's } \\
\text { method }\end{array}$ & $\begin{array}{c}\text { Density } \\
\text { measurement }\end{array}$ \\
\hline Laurylcarnitine & 570 & $553 \pm 5 \%$ \\
Myristylcarnitine & 625 & $614 \pm 5 \%$ \\
Palmitylcarnitine & 680 & $665 \pm 5 \%$ \\
\hline
\end{tabular}

observed pK decreases as the degree of protonation increases, and the effect of added $\mathrm{KCl}$ is to reduce the $\mathrm{pK}$ change at any value of $\beta$. Table II also shows that at any value of $\beta$ and concentration of $\mathrm{KCl}$ the $\mathrm{pK}$ values of the three acylcarnitines are relatively constant. The effects of ionic strength and $\beta$ on the surface potential of the micelle, computed from Eq. [1], are also illustrated in Fig. 1.

Partial Molecular Volume. The partial molecular volumes of micellar lauryl-, myristyl-, and palmitylcarnitine, exclusive of their counterions, as calculated by Traube's method and as determined experimentally, are shown in Table III. There was no detectable change in the experimentally determined values with ionization of the micelle or with increasing $\mathrm{KCl}$ concentration, confirming the conclusions of Stigter in this regard (20).

Light Scattering. In Fig. 2 values of $H\left(C-C_{0}\right) / \tau-\tau_{0}$ (where $H$ is the Debye 
constant (21); $\tau$ and $\tau_{0}$ are, respectively, the turbidities of acylcarnitine solutions of concentrations $C$ and $C_{0}$, the critical micelle concentration) are plotted against $C-C_{0}$ for

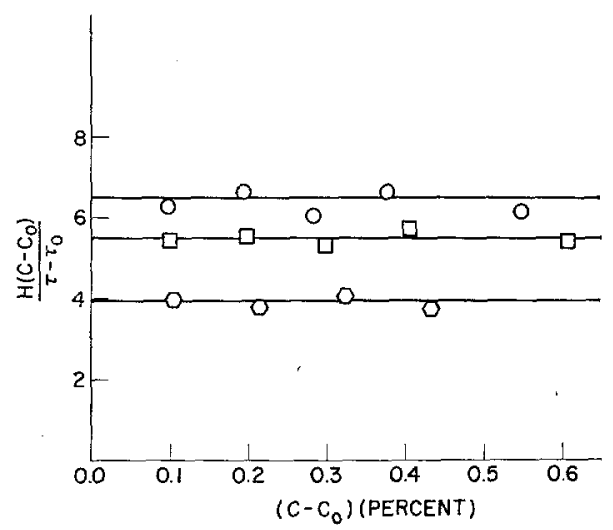

FIG. 2. Light-scattering data for cationic $(\beta$ $=$ 1.0) lauryl-, myristyl-, and palmitylcarnitine in $0.10 \mathrm{M} \mathrm{KCl}$. O : laurylcarnitine; $\square$ : myristylcarnitine; $\bigcirc$ : palmitylearnitine laurylcarnitine, myristylcarnitine, and palmitylcarnitine in $0.10 \mathrm{M} \mathrm{KCl}$ at $\beta=1.0$. Additional data were obtained for these compounds in $0.10 \mathrm{M}$ and $0.20 \mathrm{M} \mathrm{KCl}$ all at $\beta=$ $0.25,0.50,0.75$, and 1.00 . The micelle aggregation numbers in $\mathrm{KCl}$ solutions determined from the reciprocal of the intercepts of these graphs (21), all of which showed little or no slope, are listed in Table IV. These values have not been corrected for nonideal behavior as shown by Huisman (22) and would be expected to be about $10 \%-15 \%$ higher if corrected, especially at higher degrees of ionization. In all cases the micelle size decreases as the degree of ionization and the alkyl chain length increase.

Calculations of the Micelle Aggregation Number from Potentiometric Titration Data. The values of $n$ calculated by Eqs. [11] through [14] for myristylcarnitine in $0.10 \mathrm{M}$ $\mathrm{KCl}$ are shown in Fig. 3 along with the values determined by light scattering. It can

TABLE IV

Comparison of Potentiometric and Light-Scattering Data for Laurylcarnitine, Mrristylgarnitine, and Palmitylcarnitine

\begin{tabular}{|c|c|c|c|c|}
\hline \multirow{2}{*}{ Surfactant } & \multirow{2}{*}{ Solvent } & \multirow{2}{*}{$\beta$} & \multicolumn{2}{|c|}{ Micellar aggregation number } \\
\hline & & & Potentiometric data & Light-scattering data \\
\hline \multirow[t]{8}{*}{ Laurylcarnitine } & \multirow{4}{*}{$0.10 M \mathrm{KCl}$} & 0.25 & 72 & 60 \\
\hline & & 0.50 & 65 & 55 \\
\hline & & 0.75 & 55 & 48 \\
\hline & & 1.00 & 40 & 40 \\
\hline & \multirow[t]{4}{*}{$0.20 M \mathrm{KCl}$} & 0.25 & 77 & 74 \\
\hline & & 0.50 & 77 & 66 \\
\hline & & 0.75 & 65 & 60 \\
\hline & & 1.00 & 48 & 42 \\
\hline \multirow[t]{8}{*}{ Myristylcarnitine } & \multirow[t]{4}{*}{$0.10 M \mathrm{KCI}$} & 0.25 & 74 & 68 \\
\hline & & 0.50 & 55 & 55 \\
\hline & & 0.75 & 47 & 48 \\
\hline & & 1.00 & 37 & 44 \\
\hline & \multirow[t]{4}{*}{$0.20 M \mathrm{KCl}$} & 0.25 & 89 & 82 \\
\hline & & 0.50 & 86 & 78 \\
\hline & & 0.75 & 73 & 60 \\
\hline & & 1.00 & 53 & 58 \\
\hline \multirow[t]{8}{*}{ Palmitylcarnitine } & \multirow[t]{4}{*}{$0.10 M \mathrm{KCl}$} & 0.25 & 96 & 102 \\
\hline & & 0.50 & 66 & 87 \\
\hline & & 0.75 & 50 & 63 \\
\hline & & 1.00 & 40 & 58 \\
\hline & \multirow[t]{4}{*}{$0.20 M \mathrm{KCl}$} & 0.25 & 110 & 109 \\
\hline & & 0.50 & 96 & 100 \\
\hline & & 0.75 & 77 & 85 \\
\hline & & 1.00 & 60 & 79 \\
\hline
\end{tabular}




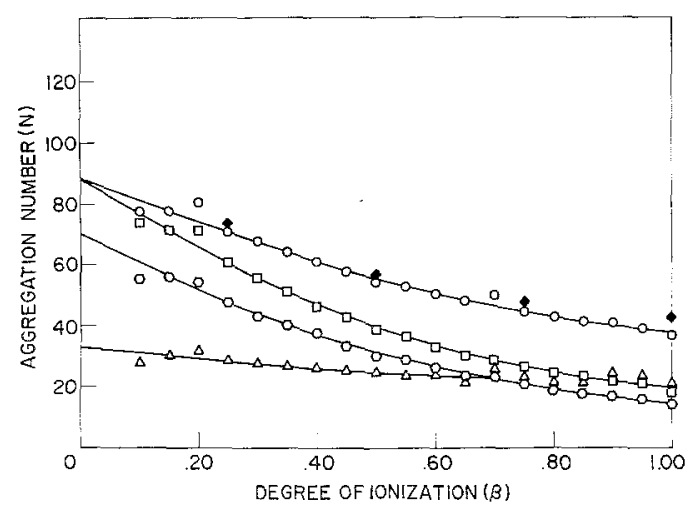

FIg. 3. Comparison of myristylcarnitine micelle radius determined by potentiometry and by light scattering. $\triangle$ : flat plate approximation; $\square$ : first Debye-Hückel approximation; $\square$ : second DebyeHückel approximation; $O$ : empirical equation of Loeb et al.; : light scattering

be seen from this figure that the treatment utilizing the empirical equation of Loeb and coworkers gives the best agreement between the potentiometric and light-scattering data. Since this was true for all systems studied only the values calculated from Eq. [13] are compared with those from light scattering in Table IV.

\section{DISCUSSION}

Figure 3 illustrates quite clearly that the flat plate and Debye-Hückel treatments do not describe the electrical properties of acylcarnitine micelles as well as the treatment which utilizes the empirical formulas of Loeb et al. (6). The flat plate approximation is valid only at very high values of $\kappa b$, i.e., when the thickness of the electrical double layer is very small compared to the radius of the particle. This is obviously not the case for a micelle but would be the case for a large emulsion droplet.

At low degrees of ionization where the surface potential is much less than $25 \mathrm{mV}$ (see Fig. 3) the first Debye-Hückel approximation gives values of $n$ which are in good agreement with those obtained through the Loeb treatment. This would be expected since both consider point charged electrolytes surrounding a spherical particle. At high values of $\beta$ the agreement between the two treatments is rather poor, indicating the importance of the higher order terms ignored in the Debye-Hückel approximation.

It might be expected intuitively that the second Debye-Hückel approximation, which accounts for the finite size of the electrolyte ions, would be more correct than the Loeb treatment, at least at low potentials where the former is mathematically valid. The better agreement found by considering electrolytes as point charges, however, can be understood if the model of the micelle, described earlier, is modified to account for the fact that the ionic groups protrude out of the hydrocarbon core of the micelle, as proposed by Stigter and Mysels (23) and Stigter (24). Mathematically this is almost equivalent to the model of a "smooth" micelle surrounded by point charge electrolyte ions, which is the model used in the treatment of Loeb and coworkers. Whatever the exact model, the good agreement between the point charge model calculations and the light-scattering data suggests that this treatment leads to a reasonable approximation of acylcarnitine micelle behavior.

In the determination of the micelle size from potentiometric titration data there are three assumptions which warrant further comment. These are the use of a smeared charge model for the micelle; the use of the dielectric constant of the solvent; and the assumption that electrolyte ions are not bound to the micelle.

Although the net charge of a particle such as a micelle is obviously not spread evenly over its surface, it can be implied from Tanford (5) that the discrete charge model is mathematically equivalent to a smeared charge model, provided that the discrete charges are distributed more or less uniformly. Since the groups on a micelle surface are free to arrange themselves in any manner, it is probably safe to assume that the similarly charged groups of the micelle in their lowest energy state will be as far apart as is consistent with the geometry of the micelle, and will, therefore, be uniformly distributed over the surface. This is especially true for a micelle containing a zwitterionic surfactant such as acylcarnitine. This is supported by a large number of studies on zwitterionic surfactants (26-30) which indicate that these compounds tend to be oriented at interfaces 
with the line connecting the two charged portions lying parallel to the interface.

The dielectric constant in the electrical double layer has been the subject of many papers (31-34), and it is generally agreed that the use of the bulk value does not introduce serious errors into the calculations. If the value of the dielectric constant at the site of the titratable group differs from its bulk value, by virtue of its position in the double layer and its proximity to a region of low dielectric constant (35), then the intrinsic dissociation constant of that group would be altered. If this is the case, the experimentally observed pG might be the net result of a residual positive charge near the carboxyl group which lowers the pK and of the effective dielectric constant around the group which raises the pK. The validity of the treatments used in this paper, however, does not depend on the pK at $\beta=0$ being the true intrinsic $\mathrm{pK}$; all that is required is that the value does not change with the degree of ionization of the micelle.

The fact that electrolyte ions are not bound to the micelle is verified by the independence of the pG on the concentration of $\mathrm{KCl}$ in the solution (36). It seems safe to assume that this is the case at any value of $\beta$ and that the counterions held in the vicinity of the positive micelle surface are held by purely electrostatic forces.

Application to Other-Systems. The raw titration data of Vochten et al. (37), for sapabolin, a weak acid, were found to give the same type of titration curve as described by Fig. 2 and to have an intercept of 4.85 . With the use of the partial molecular volume calculated from their reported specific volume of sapabolin and its molecular weight, the calculations described in this paper were performed and gave the radius and aggregation number of the unbydrated charged particle as $13.2 \AA$ and 5 . This is in excellent agreement with the value of $12.5 \AA$ for the radius and 5 for the aggregation number found by Vochten and coworkers by diffusion and light-scattering measurements. With only slight modification, therefore, it seems that equations used in this paper would be applicable to the titration of many other systems including mixed micelles and substances solubilized in micelles (38).

\section{SUMMARY AND CONCLUSIONS}

The $\mathrm{pK}$ values of acylcarnitines in the micellar state are strongly dependent on the degree of ionization of the micelle and the ionic strength of the solution but not on the chain length of the acyl-moiety.

The surface potential of acylcarnitine micelles at any degree of protonation can be calculated from the experimentally determined $\mathrm{pK}$ at that degree of protonation and the pK of the zwitterionic micelle. The surface potentials of the micelles can be used to determine their surface charge densities according to several mathematical treatments. For each treatment the calculated surface charge density and the partial molar volume of the surfactant in the micellar state can be used to calculate the radius and aggregation number of the micelle.

Comparison of these calculations with light-scattering data indicates that if the empirical equation of Loeb, Wiersema, and Overbeek is used to relate surface potential and surface charge density, the agreement between the micelle sizes determined by the two experiments is very good.

\section{REFERENCES}

1. Yalkowsky, S., and Zografi, G., J. Pharm. Sci. 59, 798 (1970).

2. Tokiwa, F., and Ohki, K., J. Phys. Chem. 70, 3437 (1966).

3. Tokiwa, F., ANd OhKi, K., Bull. Chem. Soc. (Jap.) 41, 2828 (1968).

4. Tokiwa, F., and Oнкi, K., J. Phys. Chem. 71, 1824 (1966).

5. Tanford, C., "Physical Chemistry of Macromolecules," pp 475-481. Wiley, New York, 1961.

6. Loeb, A. L., Overbeek, J. Th. G., and Wrersema, P. H., "The Electrical Double Layer around a Spherical Colloid Particle," M.I.T. Press, Cambridge, Massachusetts, 1961.

7. Adamson, A., "Physical Chemistry of Surfaces," Interscience, New York, 1960.

8. Tokrwa, F., and OHKI, K., Kolloid-Z. $Z$. Polym. 223, 135 (1967).

9. Mukerjee, P., Advan. Colloid Interface. Sci. 1, 241 (1967).

10. Ziegler, J. H., Bruchner, P., And Binon, F., J. Org. Chem. 32, 3989 (1967).

11. Albert, A., and Sergeant, E. P., "Ionization Constants of Acids and Bases." Wiley, New York, 1962. 
12. Traube, J., Sammlung Chem. u. Chem. Tech. Vortrage 4, 255 (1899).

13. Cohn, E. J., and Edsall, J. T., "Proteins, Amino Acids and Peptides." Reinhold, New York, 1943.

14. Cohn, E. J., McMerkin, T. L., Edsall, J. T., and Blanchard, M. H., J. Amer. Chem. Soc. 56, $784(1934)$.

15. LaL, H., J. Colloid Sci. 8, 414 (1953).

16. Mukerjee, P., J. Phys. Chem. 66, 1733 (1962).

17. Atwood, D., Elworthy, P. H., and Kayne, S. B., J. Pharm. Pharmacol., 21, 620 (1969).

18. Schoт, H., J. Phys. Chem. 70, 2966 (1966).

19. Katchalsky, A., Shavit, N., And Eisenberg, H., J. Polymer Sci. 13, 69 (1954).

20. Stigter, P., J. Colloid Interface. Sci. 23, 379 (1967).

21. Deвy, P., Ann. N. Y. Acad. Sci. 25, 575 (1949).

22. Hursman, F., Koninkl. Ned. Akad. Wetenschap. Proc. Ser. B 67, 367 (1964).

23. Stigter, D., and Mrsels, K. J., J. Phys. Chem. 59, 45 (1955).

24. Stigter, D., J. Phys. Chem. 68, 3603 (1964).

25. Haydon, D. A., Proc. Roy. Soc. Ser, A 258, 319 (1960).

26. Standish, M. M., And Permica, B. A., Trans. Faraday Soc. 64, 1113 (1968).
27. Beckett, A. H., and Woodward, R. J., $J$. Pharm. Pharmacol. 15, 442 (1963).

28. Hanai, T., Haydon, D. A., and Taylor, J., J. Theor. Biol. 9, 278 (1965).

29. Joos, P., And Ruyssen, R., Chem. Phys. Lipids 3, 83 (1969).

30. PethicA, B. A., "Surface Activity and the Microbial Cell," p 83. S.C.I. Monograph No. 19 (1965).

31. Wiersema, P. H., Loeb, A. L., and Overbeek, J. TH. G., J. Colloid and Interface. Sci. 22, 78 (1966).

32. Lyklema, J. J., and Orerbeek, J. Th. G., J. Colloid Sci. 16, 501 (1963).

33. Haydon, D. A., Rec. Progr. Surface Sci. 1, 94 (1964).

34. Stigter, D., and Overbeek, J. Th. G., Proc. Intern. Congr. Surface Activity $2 n d$ London 195\%, p 311.

35. MukerJee, P., and Desar, N. R., Nature 223, 1056 (1969).

36. Scatchard, G., and Black, E. S., J. Phys. and Colloid Chem. 53, 88 (1949).

37. Vochten, R., Joos, P., and Ruyssen, R., Bull. Soc. Chim. Belges 77, 473 (1968).

38. Mukerjee, P., and Banerjee, K., $J$. Phys. Chem. 68, 3567 (1964). 